Mathematical Modelling AND ANALysis

Volume 17 Number 2, April 2012, 251-270

http://dx.doi.org/10.3846/13926292.2012.662659

(c) Vilnius Gediminas Technical University, 2012
Publisher: Taylor\&Francis and VGTU

http://www.tandfonline.com/TMMA

Print ISSN: 1392-6292

Online ISSN: 1648-3510

\title{
Numerical Experiments with Single Mode Gyrotron Equations*
}

\author{
Andrejs Reinfelds ${ }^{a, b}$, Olgerts Dumbrajs ${ }^{c}$, Harijs Kalis $^{a, b}$, \\ Jānis Cepītis ${ }^{a, b}$ and Dana Constantinescu ${ }^{d}$ \\ ${ }^{a}$ Faculty of Physics and Mathematics, University of Latvia \\ Zellu iela 8, LV-1002 Rīga, Latvia \\ ${ }^{b}$ Institute of Mathematics and Computer Science of University of Latvia \\ Raiņa bulvāris 29, LV-1459 Rīga, Latvia \\ ${ }^{c}$ Institute of Solid State Physics of University of Latvia \\ Ķengaraga iela 8, LV-1063 Rīga, Latvia \\ ${ }^{d}$ Faculty of Exact Sciences, University of Craiova \\ A.I. Cuza Street 13, 200585 Craiova, Romania \\ E-mail(corresp.): reinf@latnet.lv \\ E-mail: olgerts.dumbrajs@lu.lv; harijs.kalis@lu.lv \\ E-mail: janis.cepitis@lu.lv; dconsta@yahoo.com
}

Received October 10, 2011; revised January 16, 2012; published online April 1, 2012

\begin{abstract}
Gyrotrons are microwave sources whose operation is based on the stimulated cyclotron radiation of electrons oscillating in a static magnetic field. This process is described by the system of two complex differential equations: nonlinear first order ordinary differential equation with parameter (averaged equation of electron motion) and second order partial differential equation for high frequency field ( $\mathrm{RF}$ field) in resonator (Schrödinger type equation for the wave amplitude).

The stationary problem of the single mode gyrotron equation in short time interval with real initial conditions was numerically examined in our earlier work. In this paper we consider the stationary and nonstationary problems in large time interval with complex oscillating initial conditions.

We use the implicit finite difference schemes and the method of lines realized with MATLAB. Two versions of gyrotron equation are investigated. We consider different methods for modelling new and old versions of the gyrotron equations. The main physical result is the possibility to determine the maximal value of the wave amplitude and the electron efficiency coefficient.
\end{abstract}

Keywords: finite difference scheme, gyrotron equation, method of lines, oscillation of solution.

AMS Subject Classification: 65N06; 65N22; 65N40; 65Z05.

\footnotetext{
* This work was supported by the grants 09.1220 and 09.1572 of the Latvian Council of Science and by the ESF Project 2009/0223/1DP/1.1.1.2.0/09/APIA/VIAA/008.
} 


\section{Introduction}

Temporal evolution of the amplitude of nonstationary gyrotron oscillations can be described by the following system of two partial differential equations [1, $15,16,17]$ (old version):

$$
\left\{\begin{array}{l}
\frac{\partial p}{\partial x}+\mathrm{i}\left(\Delta+|p|^{2}-1\right) p=\mathrm{i} f(t, x), \\
\frac{\partial^{2} f}{\partial x^{2}}-\mathrm{i} \frac{\partial f}{\partial t}+\delta f=I\langle p\rangle
\end{array}\right.
$$

where $\mathrm{i}=\sqrt{-1}$ is the imaginary unit, $x \in[0, L]$ and $t \in\left[0, t_{f}\right]$ are the normalized axial and temporal coordinates, $L$ is the exit from the interaction space, $t_{f}$ is the final time, $p=p\left(t, x, \theta_{0}\right)$ is the dimensionless complex transverse orbital momentum of the electron with the initial angle $\theta_{0}, 0 \leq \theta_{0}<2 \pi$, the complex function $f=f(t, x)$ is the normalized amplitude of RF field in a resonator, $\Delta$ is the cyclotron resonance mismatch (real constant), $\delta$ is the frequency mismatch, $I$ is the dimensionless beam current parameter. Here at $t=0$ the initial condition $f_{0}(x)=f(0, x)$ is given in the form [15] of a complex function, $\langle p\rangle=\frac{1}{2 \pi} \int_{0}^{2 \pi} p\left(t, x, \theta_{0}\right) \mathrm{d} \theta_{0}$ is the averaged value of $p$.

The new version [5] of these equations reads:

$$
\left\{\begin{array}{c}
\frac{\partial p}{\partial x}+\mathrm{i}\left(\Delta+|p|^{2}-1-g_{b}(x)\right) p=\mathrm{i} f(t, x) \\
\frac{\partial^{2} f}{\partial x^{2}}-\mathrm{i}\left(1+\delta_{\omega}\right) \frac{\partial f}{\partial t}+\left(1+0.5\left(\delta_{\omega}+g_{c}(x)\right)\right) g_{d}(x) f \\
=I\left(1+g_{c}(x)\right)^{2}\left(1+\delta_{\omega}\right)\langle p\rangle
\end{array}\right.
$$

where $g_{b}(x), g_{c}(x)$ and $g_{d}(x)$ are given empirical functions, $\delta_{\omega}=0.046565$, $I=0.002249, \Delta=0.538374$. The new version of equations takes into account two physical effects:

1. Dependence of the electron relativistic factor on the axial coordinate;

2. Dependence of the magnetic field on the axial coordinate (magnetic field tapering).

Usually these dependencies are weak and are ignored. In such a case the old version of equations can be used. The difficulties arise in solving the nonstationary problem $(1.2)$ for large time interval $t \in(0,1000)$ with the oscillating complex initial function $f_{0}(x)=f(0, x)$. The graphs of the initial functions $\operatorname{Re}\left(f_{0}(x)\right), \operatorname{Im}\left(f_{0}(x)\right),\left|f_{0}(x)\right|$ and auxiliary functions $g_{b}(x), g_{c}(x), g_{d}(x)$ for $L=113.7$ are shown in Fig. 1 and Fig. 2. In the calculations we use a uniform spatial grid with 536 mesh points. In order to reduce numerical computations we also decrease the number of grid points to 268 deleting every second $f_{0}(x)$ grid point. Similarly we obtain the grid with 134 mesh points. The equation of electron momentum has to be supplemented by the standard initial condition with the parameter $\theta_{0}$

$$
p\left(t, 0, \theta_{0}\right)=\exp \left(\mathrm{i} \theta_{0}\right)
$$




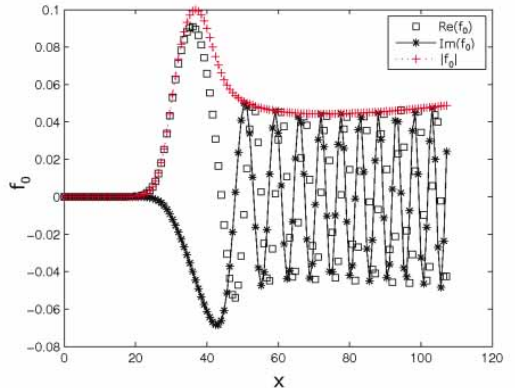

Figure 1. Complex initial function $f_{0}$.

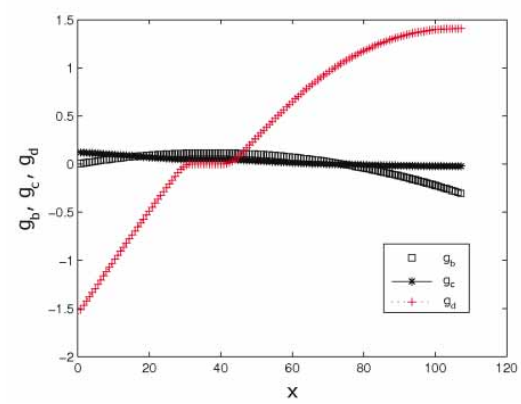

Figure 2. Auxiliary functions $g_{b}, g_{c}, g_{d}$.

and the equation of RF field by the boundary conditions at the entrance and exit of the interaction space

$$
f(t, 0)=0, \quad \frac{\partial f}{\partial x}(t, L)=-\mathrm{i} \gamma f(t, L),
$$

where $\gamma$ is a positive parameter $(L=113.7, \gamma=1.193498)$.

$$
\eta=1-\frac{1}{2 \pi} \int_{0}^{2 \pi}\left|p\left(t, L, \theta_{0}\right)\right|^{2} \mathrm{~d} \theta_{0}
$$

is the electron perpendicular efficiency which describes the transfer of the electron orbital momentum from the beam to RF.

Using implicit finite difference scheme with constant time step $\tau$ in order to solve the initial boundary value problem (1.2)-(1.4) (new version) we have difficulties to choose the value $\tau$. The maximal number of mesh points (536) was chosen for the spatial variable $x$ and computations were performed for $\left(t_{f} \approx 1000\right)$. This was repeated with smaller time steps. Against all the expectations that oscillations of amplitude will vanish with decreasing temporal step length, we observe that oscillations of the function $|f(t, x)|$ increase both in time and in space (see, Fig. 3). So, the question arises about the origin of these oscillations: are they physical or a product of numerical effects? In order to answer this question we have discretized only the space leaving the time continuous (the method of lines) and have solved the corresponding systems of differential equations with the build-in MATLAB solvers with automatic time step selection. This was done for both versions of gyrotron equations.

Our goal in this work is the numerical analysis of the stationary and nonstationary equations (1.1) and (1.2). We can write these equations in the following form:

$$
\left\{\begin{array}{l}
\frac{\partial p}{\partial x}=F_{1}(p, f), \quad p\left(t, 0, \theta_{0}\right)=\exp \left(\mathrm{i} \theta_{0}\right) \\
\frac{\partial f}{\partial t}=\mathfrak{L} f+F_{2}(\langle p\rangle) \\
f(t, 0)=0, \quad \frac{\partial f}{\partial x}(t, L)=-\mathrm{i} \gamma f(t, L), \quad f(0, x)=f_{0}(x)
\end{array}\right.
$$




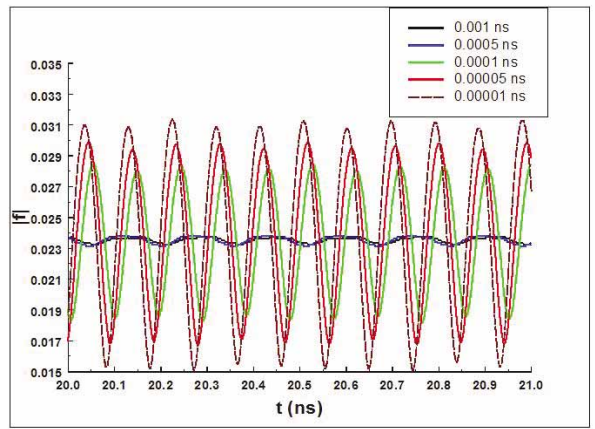

Figure 3. $|f|$ as a function of time $t$ for different dimensional temporal steps. Here the dimensionless time scale is equal $50, \delta_{\omega}=0.046565, I=0.002249, \Delta=0.538374$, $\gamma=1.193498, L=113.7$.

where $F_{1}(p, f)$ is a nonlinear complex function of $p, f, x, t, \theta_{0}, F_{2}(\langle p\rangle)$ is complex function of $\langle p\rangle, x, t, \mathfrak{L}$ is the second order linear differential operator with variable coefficients. For equations (1.1) we have:

$$
\begin{aligned}
& F_{1}(p, f)=-\mathrm{i}\left(\Delta+|p|^{2}-1\right) p+\mathrm{i} f(t, x), \\
& \mathfrak{L} f=-\mathrm{i}\left(\frac{\partial^{2} f}{\partial x^{2}}+\delta f\right), \\
& F_{2}(\langle p\rangle)=\mathrm{i} I\langle p\rangle
\end{aligned}
$$

and for equations (1.2):

$$
\begin{aligned}
& F_{1}(p, f)=-\mathrm{i}\left(\Delta+|p|^{2}-1-g_{b}(x)\right) p+\mathrm{i} f(t, x), \\
& \mathfrak{L} f=-\mathrm{i}\left(\frac{\partial^{2} f}{\partial x^{2}}+\left(1+0.5\left(\delta_{\omega}+g_{c}(x)\right)\right) g_{d}(x) f\right)\left(1+\delta_{\omega}\right)^{-1}, \\
& F_{2}(\langle p\rangle)=\mathrm{i} I\left(1+g_{c}(x)\right)^{2}\langle p\rangle .
\end{aligned}
$$

The expressions (1.7) follow from (1.8) in the case of $g_{b}(x)=g_{c}(x)=0, g_{d}=\delta$, $\delta_{\omega}=0$. For numerical analysis we consider separately equations (1.1) and (1.2). The main attention was paid to the second RF field equation because the first nonlinear momentum equation can be solved numerically with the RungeKutta method. Let us note that the model equation

$$
\frac{\mathrm{d} p}{\mathrm{~d} x}+\mathrm{i}\left(\Delta+|p|^{2}-1\right) p=\mathrm{i} F_{0} \exp (-\mathrm{i} \lambda \pi x / L),
$$

which describes the case of an infinitely long idealized resonator in which RF field is represented by a propagating wave was analyzed using qualitative methods in $[2,12,13]$. Here $F_{0}$ and $\lambda$ are the RF amplitude and frequency. This equation can be used also for equation $(1.2)$ because $g_{b}(x) \approx 0$. The numerical analysis of (1.9) for $L=15$ shows that the electron perpendicular efficiency $\eta$ as a function of parameters $F_{0}, \Delta_{\lambda}=\Delta-\lambda \pi / L$ considerably oscillates and the maximal value $\eta=0.3262$ is obtained for $F_{0}=0.25$ and $\Delta_{\lambda}=0.83$. 


\section{Conservation Equations and Some Estimates for the Continuous Problem}

The RF - field equation for fixed value $\langle p\rangle$ is $1 \mathrm{D}$ linear time-dependent Schrödinger type equation in bounded domain with boundary conditions (1.4). A review of different boundary conditions for linear and nonlinear Schrödinger equations in unbounded domain is given in [3]. There are many papers devoted to theoretical and numerical studies $[4,8,9,14,18]$. Using ideas of [8] where absorbing boundary conditions are considered, we obtain some estimates for microwave energy

$$
W(t)=\int_{0}^{L} f(t, x) f^{*}(t, x) \mathrm{d} x=\int_{0}^{L}|f(t, x)|^{2} \mathrm{~d} x
$$

stored in the resonator, where $f^{*}(t, x)$ is complex conjugate value of $f(t, x)$ and $|f(t, x)|^{2}=f(t, x) f^{*}(t, x)$. From the first complex and complex conjugated equation of (1.6) we obtain the expression

$$
\frac{\partial}{\partial x}|p|^{2}=\frac{\partial p}{\partial x} p^{*}+p \frac{\partial p^{*}}{\partial x}=2 \operatorname{Im}\left(p f^{*}\right) .
$$

Similarly, from the second complex and complex conjugated equation of (1.6) using boundary conditions (1.4) and integrating with respect to $x \in[0, L]$ the diffraction term, we obtain the conservation equation

$$
\frac{\mathrm{d} W}{\mathrm{~d} t}+2 \delta_{1} \gamma|f(t, L)|^{2}=-2 I \operatorname{Im} \int_{0}^{L} g_{2}(x) f^{*}\langle p\rangle \mathrm{d} x,
$$

where $\delta_{1}=1 /\left(1+\delta_{\omega}\right), g_{2}(x)=\left(1+g_{c}(x)\right)^{2}$. By integrating $(2.1)$ with respect to $x \in[0, L]$ and $\theta_{0} \in[0,2 \pi]$, with and taking into account initial conditions (1.3) and expression (1.5), we obtain

$$
\eta(t)=-2 \operatorname{Im} \int_{0}^{L} f^{*}\langle p\rangle \mathrm{d} x .
$$

Then from (2.2) it follows, that

$$
I \eta=\frac{\mathrm{d} W}{\mathrm{~d} t}+2 \delta_{1} \gamma|f(t, L)|^{2}-2 I \operatorname{Im} \int_{0}^{L}\left(1-g_{2}(x)\right) f^{*}\langle p\rangle \mathrm{d} x .
$$

For the equation (1.1) from (2.4) follows the law of conservation of power in the system "electron beam + microwave field"

$$
I \eta=\frac{\mathrm{d} W}{\mathrm{~d} t}+P_{r a d}
$$

where $P_{\text {rad }}=2 \gamma|f(t, L)|^{2}$ is the microwave power radiated through the output cross-section of the resonator $x=L[6]$.

If $g_{2}(x) \not \equiv 1$, then from $(2.1)$ by integrating with respect to $\theta_{0} \in[0,2 \pi]$ we obtain

$$
2 \operatorname{Im}\left(f^{*}\langle p\rangle\right)=\frac{1}{2 \pi} \frac{\partial}{\partial x} \int_{0}^{2 \pi}\left|p\left(t, x, \theta_{0}\right)\right|^{2} \mathrm{~d} \theta_{0} .
$$


By integrating by parts with respect to $x \in[0, L]$ and using (1.3), (1.5), we obtain

$$
\begin{aligned}
2 \operatorname{Im} \int_{0}^{L} & g_{2}(x) f^{*}\langle p\rangle \mathrm{d} x=\frac{1}{2 \pi} \int_{0}^{L} g_{2}(x) \frac{\partial}{\partial x} \int_{0}^{2 \pi}\left|p\left(t, x, \theta_{0}\right)\right|^{2} \mathrm{~d} \theta_{0} \mathrm{~d} x \\
= & -\frac{1}{2 \pi} \int_{0}^{L} g_{2}^{\prime}(x) \int_{0}^{2 \pi}\left|p\left(t, x, \theta_{0}\right)\right|^{2} \mathrm{~d} \theta \mathrm{d} x+g_{2}(L)(1-\eta)-g_{2}(0) .
\end{aligned}
$$

Therefore from (2.2) the conservation equation follows

$$
\frac{\mathrm{d} W}{\mathrm{~d} t}+2 \delta_{1} \gamma|f(t, L)|^{2}-I \int_{0}^{L} g_{2}^{\prime}(x)\left\langle|p|^{2}\right\rangle \mathrm{d} x=I\left(g_{2}(0)-g_{2}(L)+\eta g_{2}(L)\right),
$$

where $g_{2}^{\prime}(x)=2\left(1+g_{c}(x)\right) g_{c}^{\prime}(x)<0, g_{c}(L)=-0.021892, g_{2}(0)-g_{2}(L) \approx 0,29$. If $I=0$, then the microwave energy is not increased in time

$$
W(t)=W(0)-2 \delta_{1} \gamma \int_{0}^{t}|f(t, L)|^{2} \mathrm{~d} t \leq W(0) .
$$

We can similarly as in [8] obtain also the conservation equation for the function

$$
E(t)=\delta_{1} \int_{0}^{L}\left|\frac{\partial f}{\partial x}(t, x)\right|^{2} \mathrm{~d} x-\int_{0}^{L} g_{1}(x)|f(t, x)|^{2} \mathrm{~d} x
$$

where $g_{1}(x)=\frac{1+0,5\left(\delta_{\omega}+g_{c}(x)\right)}{1+\delta_{\omega}} g_{d}(x)$. From second complex and complex conjugated equation of (1.6) using boundary conditions (1.4) and integrating with respect to $x \in[0, L]$ the diffraction term, we obtain

$$
\begin{aligned}
0= & \delta_{1} \int_{0}^{L}\left(\frac{\partial f}{\partial x} \frac{\partial^{2} f^{*}}{\partial t \partial x}+\frac{\partial f^{*}}{\partial x} \frac{\partial^{2} f}{\partial t \partial x}\right) \mathrm{d} x-2 \delta_{1} \gamma \operatorname{Im}\left(f(t, L) \frac{\partial f^{*}}{\partial t}(t, L)\right) \\
& -\int_{0}^{L} g_{1}(x) \frac{\partial}{\partial t}|f(t, x)|^{2} \mathrm{~d} x+2 I \operatorname{Re} \int_{0}^{L} g_{2}(x)\langle p\rangle \frac{\partial f^{*}}{\partial t}(t, x) \mathrm{d} x
\end{aligned}
$$

or

$$
\frac{\mathrm{d} E(t)}{\mathrm{d} t}=2 \delta_{1} \gamma \operatorname{Im}\left(f(t, L) \frac{\partial f^{*}}{\partial t}(t, L)\right)-2 I \operatorname{Re} \int_{0}^{L} g_{2}(x)\langle p\rangle \frac{\partial f^{*}}{\partial t}(t, x) \mathrm{d} x .
$$

From (2.1), (2.2) we obtain a priori estimates. From (2.1) in accordance with Young's type inequality it follows that

$$
\left\langle|p|^{2}\right\rangle=1+2 \operatorname{Im} \int_{0}^{x} f^{*}\langle p\rangle \mathrm{d} x \leq 1+2 L \int_{0}^{x}|f(t, x)|^{2} \mathrm{~d} x+\frac{1}{2 L} \int_{0}^{x}|\langle p\rangle|^{2} \mathrm{~d} x
$$

for all $x \in(0, L)$. Using Jensen's inequality we get $|\langle p\rangle|^{2} \leq\left\langle|p|^{2}\right\rangle$. Therefore

$$
\int_{0}^{L}\left\langle|p|^{2}\right\rangle \mathrm{d} x \leq L+2 L^{2} \int_{0}^{L}|f(t, x)|^{2} \mathrm{~d} x+\frac{1}{2} \int_{0}^{L}\left\langle|p|^{2}\right\rangle \mathrm{d} x
$$

or

$$
\int_{0}^{L}\left\langle|p|^{2}\right\rangle \mathrm{d} x \leq 2 L+4 L^{2} \int_{0}^{L}|f(t, x)|^{2} \mathrm{~d} x
$$


From (2.2) it follows

$$
\begin{aligned}
& \frac{\mathrm{d}}{\mathrm{d} t} \int_{0}^{L}|f(t, x)|^{2} \mathrm{~d} x+2 \delta_{1} \gamma|f(t, L)|^{2} \\
& \quad \leq I \max _{x}\left|g_{2}(x)\right|\left(\int_{0}^{L}|f(t, x)|^{2} \mathrm{~d} x+\int_{0}^{L}\left\langle|p|^{2}\right\rangle \mathrm{d} x\right) .
\end{aligned}
$$

Consequently

$$
\begin{aligned}
& \frac{\mathrm{d}}{\mathrm{d} t} \int_{0}^{L}|f(t, x)|^{2} \mathrm{~d} x+2 \delta_{1} \gamma|f(t, L)|^{2} \\
& \quad \leq I \max _{x}\left|g_{2}(x)\right|\left[\left(1+4 L^{2}\right) \int_{0}^{L}|f(t, x)|^{2} \mathrm{~d} x+2 L\right] .
\end{aligned}
$$

Thus the solution $f$ does not blow up in finite time. From (2.3) we obtain the corresponding estimate

$$
|\eta(t)| \leq 2 L \int_{0}^{L}|f(t, x)|^{2} \mathrm{~d} x+\frac{1}{2 L} \int_{0}^{L}\left\langle|p|^{2}\right\rangle \mathrm{d} x \leq(4 L+1) \int_{0}^{L}|f(t, x)|^{2} \mathrm{~d} x+1 .
$$

From (2.5) it follows

$$
\begin{aligned}
& \frac{\mathrm{d}}{\mathrm{d} t} \int_{0}^{L}|f(t, x)|^{2} \mathrm{~d} x+2 \delta_{1} \gamma|f(t, L)|^{2}+\min _{x}\left(-g_{2}^{\prime}(x)\right) I \int_{0}^{L}\left\langle|p|^{2}\right\rangle \mathrm{d} x \\
& \quad \leq I\left(g_{2}(0)+g_{2}(L)(4 L+1) \int_{0}^{L}|f(t, x)|^{2} \mathrm{~d} x\right) .
\end{aligned}
$$

\section{$3 \quad$ RF Field Gyrotron Equation}

The first problem of (1.6) is the nonlinear ordinary differential equation initial value problem for complex function $p(x)$ with fixed values of $f, t$ and $\theta_{0}$. It can be solved numerically with standard methods, for example, with the RungeKutta method using MATLAB solver "ode45" at each time moment $t$ and a series of values of $\theta_{0} \in[0,2 \pi)$.

The second problem is the Schrödinger type linear partial differential equation initial-boundary value problem for a complex function $f(t, x)$ for fixed values of $\langle p\rangle$. It can be solved numerically with the method of lines with respect to $t$ and discretization in space for differential operator $\mathfrak{L}$ by means of finite difference second order approximation using MATLAB solver "ode15s" (the stiff system of ordinary differential equation) or with the implicit finite difference scheme using discretization also in time. The two problems must be solved simultaneously at each time moment.

For the approximation of the derivatives in space we use a uniform grid with the step $h$ and grid points $x_{j}=(j-1) h, j=\overline{1, M}, N h=L$, where $M=N+1$ is the number of grid points. Using these grid points we can approximate the second order derivative $d^{2} f(t, x)=\frac{\partial^{2} f}{\partial x^{2}}(t, x)$ in the equations (1.6) with the central finite difference of second order in the form

$$
d^{2} f\left(t, x_{j}\right) \approx \frac{1}{h^{2}}\left(f_{j-1}(t)-2 f_{j}(t)+f_{j+1}(t)\right), \quad j=\overline{2, N},
$$


where $f_{j}(t)=f\left(t, x_{j}\right), f_{1}(t)=0$. We consider three numerical approximations of the boundary conditions at $x=L$ for the boundary condition (1.4):

- From Taylor expansion $f_{N}=f_{M}+\mathrm{i} h \gamma f_{M}+\frac{h^{2}}{2} d^{2} f(t, L)$ and finite difference expression for $j=M$, it follows that (approximation $O\left(h^{2}\right)$ ) $d^{2} f(t, L) \approx \frac{2}{h^{2}}\left(f_{N}(t)-f_{M}(t)-\mathrm{i} h \gamma f_{M}(t)\right)$. From (1.6) and (1.8) the following ordinary differential equation at the boundary point can be written:

$$
\begin{aligned}
& \frac{\mathrm{d} f_{M}}{\mathrm{~d} t}=2 \alpha\left(-f_{N}(t)+(1+\mathrm{i} h \gamma) f_{M}(t)\right) \\
& \quad-\mathrm{i}\left(1+0.5\left(\delta_{\omega}+g_{c}(L)\right)\right) g_{d}(L)\left(1+\delta_{\omega}\right)^{-1} f_{M}(t)+\mathrm{i} I\left(1+g_{c}(L)\right)^{2}\langle p\rangle_{M},
\end{aligned}
$$

where $\alpha=\mathrm{i} /\left(h^{2}\left(1+\delta_{\omega}\right)\right)$;

- From Taylor expansion $f_{N}=f_{M}+\mathrm{i} h \gamma f_{M}+\frac{h^{2}}{2} d^{2} f(t, L)+O\left(h^{3}\right), f_{N-1}=$ $f_{M}+2 \mathrm{i} h \gamma f_{M}+\frac{4 h^{2}}{2} d^{2} f(t, L)+O\left(h^{3}\right)$ the following three-point stencil formula of second order $O\left(h^{2}\right)$ approximation can be written:

$$
4 f_{N}-f_{N-1}=(3+2 \mathrm{i} h \gamma) f_{M}
$$

- The first order $O(h)$ approximation of boundary conditions can be written in following form:

$$
f_{N}=(1+\mathrm{i} h \gamma) f_{M}
$$

We use discrete values of the parameter $\theta_{0}$ in the form $\theta_{k}=k \frac{2 \pi}{K}, k=\overline{1, K}$, where $K$ is the number of angular grid points. Let $p_{j}\left(\theta_{k}\right)=p\left(t, x_{j}, \theta_{k}\right)$ be grid function values for a fixed time $t$. Using the trapezoid rule of the quadrature formula in calculating the integral in $\langle p\rangle_{j}$ (1.6), we obtain the following expression:

$$
\langle p\rangle_{j}=\frac{1}{K} \sum_{k=1}^{K} p_{j}\left(\theta_{k}\right), \quad j=\overline{1, M} .
$$

Similarly from (1.5) we can calculate the electron efficiency

$$
\eta=1-\frac{1}{K} \sum_{k=1}^{K}\left|p_{M}\left(\theta_{k}\right)\right|^{2} .
$$

\subsection{Method of lines}

First, we consider the method of lines in solving the Schrödinger type equations (1.2) in the form of the initial value problem for the following ordinary differential equation system:

$$
\frac{\mathrm{d} \boldsymbol{f}(t)}{\mathrm{d} t}=\mathbf{A} \boldsymbol{f}(t)+\boldsymbol{G}(t), \quad \boldsymbol{f}(0)=\boldsymbol{f}_{0},
$$

where $\mathbf{A}$ is the $M$ order 3-diagonal matrix with the elements

$$
\begin{aligned}
& a_{j, j}=2 \alpha+g_{j}, \quad a_{j, j+1}=a_{j, j-1}=-\alpha, \quad j=\overline{2, N}, \\
& g_{j}=-\mathrm{i}\left(1+0.5\left(\delta_{\omega}+g_{c}\left(x_{j}\right)\right)\right) g_{d}\left(x_{j}\right)\left(1+\delta_{\omega}\right)^{-1}, \quad a_{1,1}=2 \alpha, \quad a_{1,2}=0,
\end{aligned}
$$




$$
a_{M, N}=-2 \alpha, \quad a_{M, M}=2 \alpha(1+\mathrm{i} h \gamma)+g_{M}, \quad \alpha=\mathrm{i} h^{-2},
$$

$\boldsymbol{f}(t), \boldsymbol{f}_{0}, \boldsymbol{G}(t)$ are column vectors with the elements $f_{j}(t), f_{0}\left(x_{j}\right)$ and $G_{j}(t)=$ $\mathrm{i} I\left(1+g_{c}\left(x_{j}\right)\right)^{2}\langle p\rangle_{j}, j=\overline{2, M}, f_{1}(t)=\langle p\rangle_{1}=0$.

The system of ordinary differential equations (3.4) is of $M^{\text {th }}$ order and it can be solved with the MATLAB solvers "ode15s" or "ode23t" using the mass-matrix (the first equation $f_{1}(t)=0$ is algebraic). With other solvers ("ode45", "ode113", "ode23s") we can solve the system of ordinary differential equations of the $N^{t h}$ order (without mass-matrix). We use the solver "ode15" with relative error $10^{-6}$ and without mass-matrix.

\subsection{Conservation equations and some estimates for the discrete problem}

Let us use the following finite differences operators:

$$
\partial f_{j}=\frac{f_{j+1}-f_{j}}{h}, \quad \bar{\partial} f_{j}=\frac{f_{j}-f_{j-1}}{h}, \partial \bar{\partial} f_{j}=\frac{f_{j-1}-2 f_{j}+f_{j+1}}{h^{2}}=\bar{\partial} \partial f_{j} .
$$

The system of equations (3.4) with boundary conditions (3.1) can be rewritten in the following form [8]:

$$
\begin{aligned}
& \frac{\partial f_{j}}{\partial t}=-\mathrm{i} \delta_{1} \partial \bar{\partial} f_{j}-\mathrm{i} g_{1}\left(x_{j}\right) f_{j}+\mathrm{i} I g_{2}\left(x_{j}\right)\langle p\rangle_{j}, \quad j=2, \ldots, N \\
& \frac{\partial f_{M}}{\partial t}=-\mathrm{i} \delta_{1}\left(-\frac{2}{h} \bar{\partial} f_{M}-\frac{2 \mathrm{i}}{h} \gamma f_{M}\right)-\mathrm{i} g_{1}(L) f_{j}+\mathrm{i} g_{2}(L)\langle p\rangle_{M} .
\end{aligned}
$$

From (3.6) and the central difference $\frac{\partial f}{\partial x}(t, L)=\frac{f_{M+1}-f_{N}}{2 h}+O\left(h^{2}\right)$ it follows that $-\frac{2}{h}\left(\bar{\partial} f_{M}+\mathrm{i} \gamma f_{M}\right)=\partial \bar{\partial} f_{M}$.

From complex and complex conjugated equations (3.5) and (3.6) multiplying correspondingly with $f^{*}$ and $f$ and summing, we obtain

$$
\begin{aligned}
& \frac{\partial}{\partial t}\left|f_{j}\right|^{2}=\mathrm{i} \delta_{1}\left(\partial \bar{\partial} f_{j}^{*} f_{j}-\partial \bar{\partial} f_{j} f_{j}^{*}\right)+2 I g_{2}\left(x_{j}\right) \operatorname{Im}\left(f_{j}^{*}\langle p\rangle_{j}\right), \quad j=2, \ldots, N \\
& \frac{\partial}{\partial t}\left|f_{M}\right|^{2}=\frac{2 \delta_{1}}{h}\left(\mathrm{i}\left(\bar{\partial} f_{M} f_{M}^{*}-\bar{\partial} f_{M}^{*} f_{M}\right)-2 \gamma\left|f_{M}\right|^{2}\right)-2 I g_{2}(L) \operatorname{Im}\left(f_{M}^{*}\langle p\rangle_{M}\right) .
\end{aligned}
$$

Let us introduce the discrete inner products and the norms

$$
\begin{aligned}
& (u, v)=h \sum_{j=2}^{N} u_{j} v_{j}^{*}, \quad\|u\|_{h}^{2}=(u, u) \\
& (u, v]_{h}=h \sum_{j=2}^{N} u_{j} v_{j}^{*}+\frac{h}{2} u_{M} v_{M}^{*}, \quad\|u\|_{h}^{2}=(u, u]_{h}
\end{aligned}
$$

Applying the summation by parts of the discrete diffraction operator [19], we obtain the discrete energy conservation equation

$$
\left.\frac{\partial}{\partial t} \| f \mid\right]_{h}^{2}+2 \delta_{1} \gamma\left|f_{M}\right|^{2}=-2 I \operatorname{Im}\left(g_{2} f^{*}\langle p\rangle, 1\right]_{h} .
$$


This form is analogue of (2.2) where the integral is approximated by a trapezoidal formula. For boundary conditions of first order (3.3) $\bar{\partial} f_{M}=-\mathrm{i} \gamma f_{M}$, $\bar{\partial} f_{M}^{*}=\mathrm{i} \gamma f_{M}^{*}$ it follows from (3.5)

$$
\begin{aligned}
\frac{\partial}{\partial t}\|f\|_{h}^{2} & =\delta_{1}\left(\mathrm{i}\left(f_{M} \bar{\partial} f_{M}^{*}-f_{M}^{*} \bar{\partial} f_{M}\right)-2 I \operatorname{Im}\left(g_{2} f^{*}\langle p\rangle, 1\right)_{h}\right. \\
& =-2 \delta_{1} \gamma\left|f_{M}\right|^{2}-2 I \operatorname{Im}\left(g_{2} f^{*}\langle p\rangle, 1\right)_{h},
\end{aligned}
$$

i.e., equality (3.7) conserves the norm $\|t, \cdot\|_{h}$ which differs from norm $\left.\| t, \cdot \mid\right]$ by $O(h)$.

For the boundary conditions (3.2) in the form $\bar{\partial} f_{M}=-\frac{h}{2} \partial \bar{\partial} f_{M}-\mathrm{i} \gamma f_{M}$ it follows that in (3.8)

$$
f_{M} \partial f_{M}^{*}-f_{M}^{*} \bar{\partial} f_{M}=2 \mathrm{i} \gamma\left|f_{M}\right|^{2}+\Delta_{0},
$$

where $\Delta_{0}=-\frac{h}{2}\left(f_{M} \partial \bar{\partial} f_{M}^{*}-f_{M}^{*} \partial \bar{\partial} f_{M}\right)=-h \mathrm{i} \operatorname{Im}\left(f_{M} \partial \bar{\partial} f_{M}^{*}\right) \not \equiv 0$ is the disbalance term in the conservation law (3.7). For this reason the condition (3.2) is no longer used.

Using the operator $\frac{\partial f_{j}^{*}}{\partial t} \frac{\partial f_{j}}{\partial t}-\frac{\partial f_{j}}{\partial t} \frac{\partial f_{j}^{*}}{\partial t}$ for $(3.5),(3.6)$ with boundary conditions (3.1) and taking the imaginary part, we obtain

$$
\begin{aligned}
& 0=-\delta_{1}\left(\partial \bar{\partial} f_{j}^{*} \frac{\partial f_{j}}{\partial t}+\partial \bar{\partial} f_{j} \frac{\partial f_{j}^{*}}{\partial t}\right)-g_{1}\left(x_{j}\right) \frac{\partial}{\partial t}\left|f_{j}\right|^{2}+2 I \operatorname{Re}\left(\frac{\partial f_{j}^{*}}{\partial t}\langle p\rangle_{j}\right), \\
& 0=\frac{2 \delta_{1}}{h}\left[\bar{\partial} f_{M} \frac{\partial f_{M}^{*}}{\partial t}+\bar{\partial} f_{M}^{*} \frac{\partial f_{M}}{\partial t}+2 \gamma \operatorname{Im}\left(f_{M}^{*} \frac{\partial f_{M}}{\partial t}\right)\right] \\
& -g_{1}(L) \frac{\partial}{\partial t}\left|f_{M}\right|^{2}+2 I \operatorname{Re}\left(\frac{\partial f_{M}^{*}}{\partial t}\langle p\rangle\right) .
\end{aligned}
$$

Applying the summation by parts of the discrete diffraction operator we obtain the conservation equation

$$
\frac{\mathrm{d} E_{h}}{\mathrm{~d} t}(t)=2 \delta_{1} \gamma \operatorname{Im}\left(f_{M} \frac{\partial f_{M}^{*}}{\partial t}\right)-2 I \operatorname{Re}\left(g_{2}\langle p\rangle \frac{\partial f^{*}}{\partial t}, 1\right],
$$

where $\left.E_{h}(t)=\delta_{1} \| \bar{\partial} f \mid\right]_{h}^{2}-\left(g_{1}|f|^{2}, 1\right]_{h}$ is the discrete approximation of function of $E(t)$. The (3.9) is the discrete form of the conservation equation (2.6). For boundary conditions (3.3) we have

$$
C_{M}=-\delta_{1}\left(\frac{\partial f_{M}}{\partial t} \bar{\partial} f_{M}+\frac{\partial f_{M}^{*}}{\partial t} \bar{\partial} f_{M}\right)=2 \gamma \delta_{1} \operatorname{Im}\left(f_{M} \frac{\partial f_{M}^{*}}{\partial t}\right)
$$

and in the conservation equation (3.9) the form of the norm $\|\bar{\partial} f\|_{h}$ is conserved. For boundary conditions (3.2) $C_{M}=2 \gamma \delta \operatorname{Im}\left(f_{M}, \frac{\partial f_{M}^{*}}{\partial t}\right)+\Delta_{1}$, where $\Delta_{1}=$ $h \operatorname{Re}\left(\frac{\partial f_{M}}{\partial t} \partial \bar{\partial} f_{M}^{*}\right)$ is the disbalance term in the conservation law (3.9).

Similarly, the inequality $(2.7)$ can be obtained in the discrete case when $W=\| f \mid]_{h}^{2}$.

\subsection{Implicit finite difference schemes}

For the implicit finite difference scheme we use discrete values of time $t$ in the form $t_{n}=n \tau$, where $n=0,1,2, \ldots$ is the time number and $\tau$ is the temporal 
step. Let $f_{j}^{n}=f\left(t_{n}, x_{j}\right), \boldsymbol{f}^{n}=\boldsymbol{f}\left(t_{n}\right)$. Then from (3.4) the finite difference scheme follows:

$$
\left(\boldsymbol{f}^{n+1}-\boldsymbol{f}^{n}\right) / \tau=\mathbf{A} \boldsymbol{f}^{n+1}+\boldsymbol{G}^{n}
$$

where $\boldsymbol{G}^{n}=\boldsymbol{G}\left(t^{n}\right), n=0,1, \ldots, \boldsymbol{f}^{0}=\boldsymbol{f}_{0}$. We can solve this problem with the MATLAB "slash" operator in the following matrix form:

$$
\boldsymbol{f}^{n+1}=(\mathbf{E}-\tau \mathbf{A})^{-1} \boldsymbol{F}_{A},
$$

where $\boldsymbol{F}_{A}=\boldsymbol{f}^{n}+\tau \boldsymbol{G}^{n}, n=0,1, \ldots$, is the column-vector of $M^{\text {th }}$ order.

We can also solve (3.10) with the factorization method. The finite difference scheme can be written in the form

$$
A_{j} y_{j-1}-C_{j} y_{j}+B_{j} y_{j+1}=-F_{j}, \quad j=\overline{1, M},
$$

where $A_{j}=B_{j}=-\alpha \tau, C_{j}=1-\left(2 \alpha+g_{j}\right) \tau, j=\overline{2, N}, A_{1}=F_{1}=0, B_{1}=0$, $B_{M}=0, C_{1}=1, A_{M}=-2 \alpha \tau, C_{M}=1-\left(2 \alpha(1+\mathrm{i} h \gamma)+g_{M}\right) \tau, y_{j}=f_{j}\left(t^{n+1}\right)$, $F_{j}=f_{j}\left(t^{n}\right)+\tau G_{j}\left(t^{n}\right), j=\overline{2, M}$.

We can use the finite difference scheme with the weight $\sigma \in[0,1]$ in following form:

$$
\left(\boldsymbol{f}^{n+1}-\boldsymbol{f}^{n}\right) / \tau=\mathbf{A}\left(\sigma \boldsymbol{f}^{n+1}+(1-\sigma) \boldsymbol{f}^{n}\right)+\boldsymbol{G}^{n} .
$$

Then $\boldsymbol{f}^{n+1}=(\mathbf{E}-\tau \sigma \mathbf{A})^{-1} \boldsymbol{F}_{A}$, where $\boldsymbol{F}_{A}=(\mathbf{E}+\tau(1-\sigma) \mathbf{A}) \boldsymbol{f}^{n}+\tau \boldsymbol{G}^{n}$.

\section{Discrete Spectral Problems}

In paper [11] we considered the quasi-stationary solution of the homogeneous RF field equation (1.1) $(I=0)$ with boundary conditions (1.4). Using uniform grid and numerical boundary condition with $O(h)$ we solved the spectral problem for matrix $\tilde{\mathbf{A}}=\mathbf{A} / i$ : $\tilde{\mathbf{A}} \boldsymbol{w}^{k}=\mu_{k} \boldsymbol{w}^{k}$, where $\boldsymbol{w}^{k}$ is the orthonormed eigenvector with elements $w_{j}^{k}=C_{k} \sin \left(q_{k} x_{j}\right), C_{k}^{-2}=\frac{1}{2}\left(L-\frac{h \sin \left(q_{k} L\right) \cos \left(q_{k}(L-h)\right)}{\sin \left(q_{k} h\right)}\right)$, $j=\overline{1, N-1}, \mu_{k}=4 h^{-2} \sin ^{2}\left(q_{k} h / 2\right)$ is the eigenvalue obtained from the transcendental equation $\sin \left(q_{k} L\right)=(1+\mathrm{i} h \gamma)^{-1} \sin \left(q_{k}(L-h)\right), k=\overline{1, N-1}, \delta=0$. This transcendental equation has a countable set of complex roots in the right half-plane. The roots are ordered by increasing real parts.

Table 1. The values of $A_{k}, B_{k}$ for $L=15, \gamma=2, \delta=0, I=0$.

\begin{tabular}{lllrl}
\hline$N_{0}$ & $A_{k}($ old $)$ & $B_{k}($ old $)$ & $A_{k}($ new $)$ & $B_{k}($ new $)$ \\
\hline 1 & 0.0437 & 0.0029 & -0.0232 & 0.0638 \\
2 & 0.1748 & 0.0118 & 0.2576 & 0.05530 \\
3 & 0.3933 & 0.0272 & 0.6681 & 0.0826 \\
4 & 0.6988 & 0.0497 & 1.0619 & 0.0998 \\
5 & 1.0912 & 0.0807 & 1.4713 & 0.1320 \\
6 & 1.5695 & 0.1228 & 1.9282 & 0.1894 \\
7 & 2.1318 & 0.1803 & 2.4692 & 0.2599 \\
8 & 2.7715 & 0.2627 & 3.0383 & 0.3814 \\
\hline
\end{tabular}

For RF field equation (1.2) the coefficients of operator $\mathfrak{L}$ are not constants and the analytical solution of the spectral problem does not exist. We use 
the MATLAB operator "eig" and solve the spectral problem numerically. For comparison for the first 8 eigenvalues in the paper [11] we solved the spectral problems for $L=15, \gamma=2, \delta=0, N=300$ (see Tab. 1, where $A_{k}=\operatorname{Re}\left(\mu_{k}\right)$, $\left.B_{k}=\operatorname{Im}\left(\mu_{k}\right)\right)$. The obtained values $\mu_{k}$ are in agreement within four digits with the eigenvalues in [11].

Table 2. The values of $A_{k}, B_{k}$ for $L=113.7, \gamma=1.193498, \delta=0$.

\begin{tabular}{ccccc}
\hline$N_{0}$ & $A_{k}($ old $)$ & $B_{k}($ old $)$ & $A_{k}($ new $)$ & $B_{k}($ new $)$ \\
\hline 1 & 0.0008 & 0.0000 & -1.3806 & 0.0037 \\
2 & 0.0031 & 0.0000 & -1.3152 & 0.0060 \\
3 & 0.0069 & 0.0001 & -1.2483 & 0.0078 \\
4 & 0.0122 & 0.0002 & -1.1806 & 0.0095 \\
5 & 0.0191 & 0.0003 & -1.1123 & 0.0110 \\
6 & 0.0274 & 0.0004 & -1.0436 & 0.0124 \\
7 & 0.0374 & 0.0006 & -0.9745 & 0.0138 \\
8 & 0.0488 & 0.0007 & -0.9049 & 0.0153 \\
\hline
\end{tabular}

In Tab. 2 the results for $L=113.7, \gamma=1.193498$ and $N=268$ are given. The values of $A_{k}$ are different for RF field equations (1.1) and (1.2). Comparing the eigenvalues of the RF equations (1.1) and (1.2), we see that the values of $A_{k}$ have different signs (the first values of (1.2) are negative). The results do not change much (four digits remain the same) by changing twice the spatial step $h$. In [11] we obtained the stability condition of finite difference scheme (3.10) for RF field problem (1.1) in the following form $\sigma \geq 0.5, B_{k} \geq 0$.

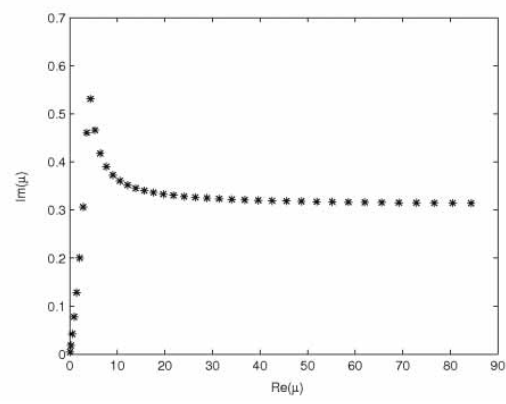

Figure 4. First 40 discrete (old) eigenvalues in the plane $(\operatorname{Re} \mu, \operatorname{Im} \mu)$, $N=300, L=15, \gamma=2, \delta=0, I=0$.

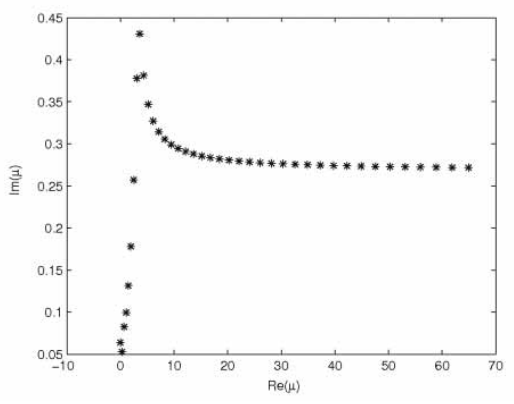

Figure 5. First 40 discrete (new) eigenvalues in the plane $(\operatorname{Re} \mu, \operatorname{Im} \mu)$, $N=300, L=15, \gamma=2, I=0$.

In Fig. 4 and Fig. 5 for $L=15, \gamma=2, h=0.1$ the first 40 eigenvalues (increasing $\operatorname{Re}\left(\mu_{k}\right)$ ) are shown in the plane $A_{k}=\operatorname{Re}\left(\mu_{k}\right), B_{k}=\operatorname{Im}\left(\mu_{k}\right)$. In Fig. 6 and Fig. $7(L=113.7, N=268, \gamma=1.193498)$ we can see that for all values $B_{k} \geq 0\left(\min \left(B_{k}\right)=-2.610^{-14}\right.$ for the new version of equations for $k>240)$ and the implicit finite difference scheme is unconditionally stable for $\sigma \geq 0.5$ independent of $\tau$. In [10] we obtained the following inequalities for different weight $\sigma$ : 


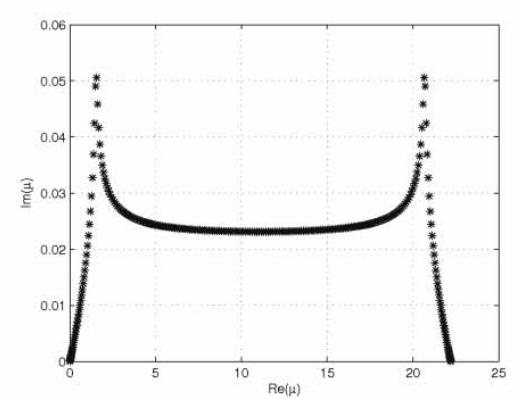

Figure 6. Discrete (old) eigenvalues in the plane $(\operatorname{Re} \mu, \operatorname{Im} \mu), N=268, L=113.7$, $\gamma=1.1934982, \delta=0, I=0$.

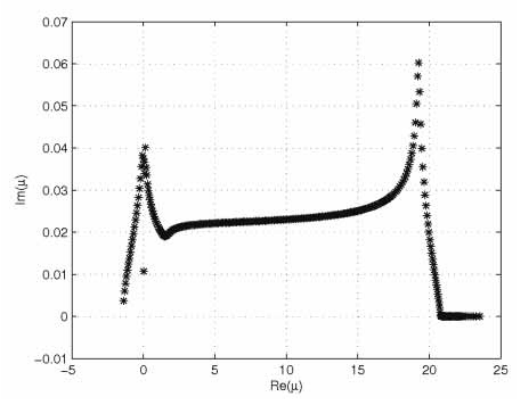

Figure 7. Discrete (new) eigenvalues in the plane $(\operatorname{Re} \mu, \operatorname{Im} \mu), N=268, L=113.7$, $\gamma=1.1934982, I=0$.

1. For $\sigma<0.5: \tau \leq 2 B_{k} /\left[(1-2 \sigma)\left(A_{k}^{2}+B_{k}^{2}\right)\right]$.

2. For $\sigma=0.5: B_{k} \geq 0$.

3. For $\sigma \in(0.5,1]: \tau \geq 2 B_{k}\left[(1-2 \sigma)\left(A_{k}^{2}+B_{k}^{2}\right)\right]$. This condition is important for $B_{k}<0$.

For $\sigma \leq 0.5$ the temporal step length $\tau$ is bounded because $B_{k}>0$ and $\max \left(A_{k}\right)$ is large (for $L=113.7 \max \left(A_{k}\right) \approx 24$ ). The maximal value $M_{\tau}$ for $\frac{2 B_{k}}{A_{k}^{2}+B_{k}^{2}}$ is 53.09 (new) and 38.63 (old).

In the calculations with increasing amplitude $f$ oscillations sometimes occur when $B_{k}<0$. In such a case the condition $\tau \geq \frac{2 B_{k}}{(1-2 \sigma)\left(A_{k}^{2}+B_{k}^{2}\right)}$ becomes important. For $(1.2)$ and $k=268(\sigma=1)$ we have $B_{N}=-3.10^{-14}, A_{N} \approx 24$ and the inequality $\tau \geq 0.510^{-6}$ is no longer important.

\section{Models of RF Field Equations}

For numerical analysis we consider the model of RF field equation for problem (1.6) with initial condition $f_{0}(x)$ and with given function $\langle p\rangle=\exp (\mathrm{i} \lambda x)$ with $\lambda=0$ and $\lambda=1$. The corresponding stationary problem is solved using the MATLAB solver "bvp4c" and cubic spline interpolation for initial complex function $f_{0}(x)$ and auxiliary functions $g_{c}(x)$ and $g_{d}(x)$.

In the stationary case from $(1.6),(1.7)$ for $\delta=0$ we obtain the test problem $f^{\prime \prime}(x)=I \exp (\mathrm{i} \lambda x), f(0)=0, f^{\prime}(L)=-\mathrm{i} \gamma f(L)$ with the following analytical solutions:

1. for $\lambda=0: f(x)=0.5 I x^{2}+C_{1} x, C_{1}=I L(1-0.5 \mathrm{i} \gamma L) /(1+\mathrm{i} \gamma L)$,

2. for $\lambda=1: f(x)=-I \exp (\mathrm{i} x)+C_{1} x+I, C_{1}=\mathrm{i} I(\exp (\mathrm{i} L)(1+\gamma)-\gamma) /(1+$ $\mathrm{i} \gamma L)$.

Using the approximation of boundary conditions of third kind (3.1), (3.2), (3.3) we obtain for $N=134 ; 268 ; 536, L=113.7$ and $I=0.002249$ the following maximal value of $|f|$ : 


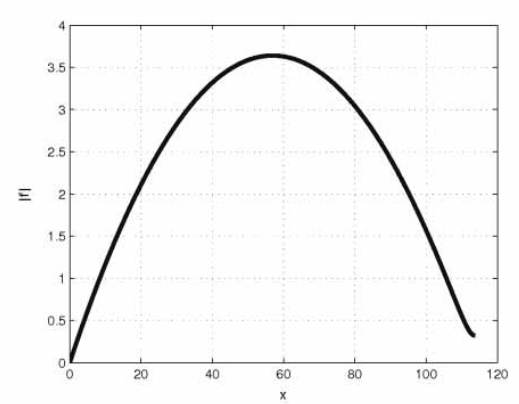

Figure 8. Stationary solution $|f|$ (old), $\lambda=0, L=113.7, N=536, \delta=0$, $I=0.002249$.

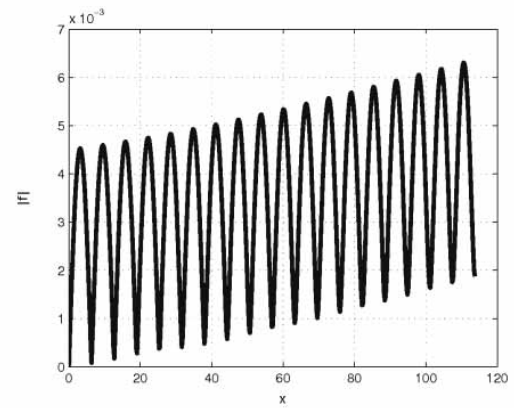

Figure 9. Stationary solution $|f|$ (old), $\lambda=1, L=113.7, N=536, \delta=0$, $I=0.002249$.

1. for $\lambda=0$ : (Schrödinger type solution, see Fig. 8) 3.6380 (analytical solution), 3.6372 for (3.3), 3.6374 for (3.2), 3.6374 for (3.1);

2. for $\lambda=1$ : (oscillating solution, see Fig. 9), $N=134$ : 0.005566 (analytical sol.), 0.005849 for (3.3), 0.006152 for $(3.2), 0.005775$ for $(3.1) . N=268$ : 0.005591 (analytical sol.), 0.005649 for $(3.3), 0.005739$ for $(3.2), 0.005641$ for (3.1), $N=536$ : 0.005608 (analytical sol.), 0.005626 for (3.3), 0.005646 for $(3.2), 0.005621$ for (3.1).

Hence, the boundary condition $(3.1) f_{N}-(1+\mathrm{i} h \gamma) f_{M}=0.5 \mathrm{i} I h^{2} \exp (\mathrm{i} \lambda L)$ is more accurate. In what follows we will use only the boundary conditions (3.1).

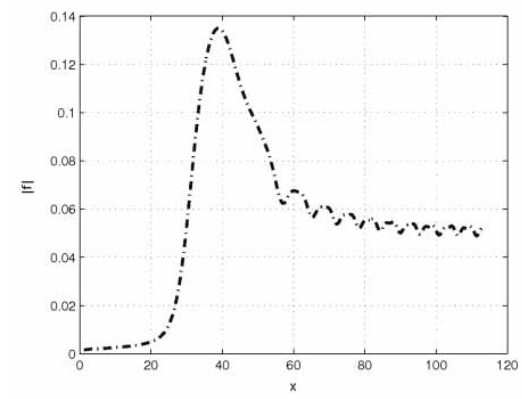

Figure 10. Stationary solution $|f|$ (new), $\lambda=0, L=113.7, N=134$.

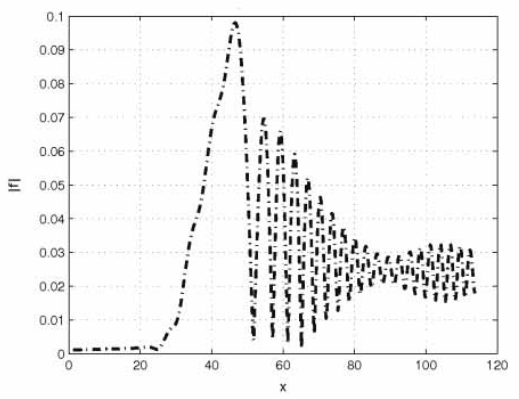

Figure 11. Stationary solution $|f|$ (new), $\lambda=1, L=113.7, N=536$.

Using the stationary model equations for (1.6), (1.8), we obtain the following maximal value of $|f|$ for different $N$ :

1. for $\lambda=0$ : $0.1342(N=134), 0.1730(N=268), 0.1750(N=536)$ (see Fig. 10),

2. for $\lambda=1: 0.0794(N=134), 0.1074(N=268), 0.0980(N=536)$ (see Fig. 11). 


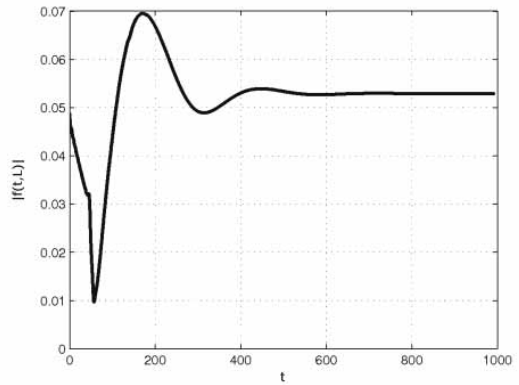

Figure 12. Dependence of $|f(t, L)|$ on $t$ (new), $\lambda=0, L=113.7, \tau=0,0496$, $h=0,42$.

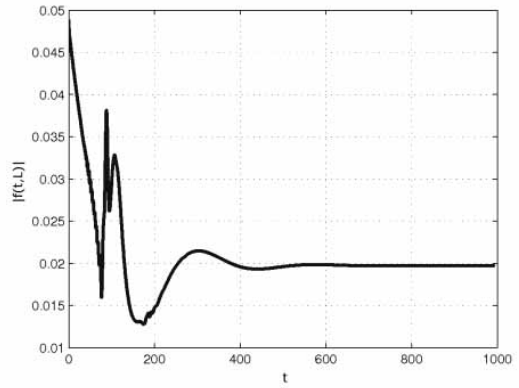

Figure 13. Dependence of $|f(t, L)|$ on $t$ (new), $\lambda=1, L=113.7, \tau=0,0496$, $h=0,42$.

For the corresponding nonstationary solution $|f(t, L)|$ for the new version of equations for $t_{f}=1000, N=536$ the temporal behaviour is shown in Fig. 12 $(\lambda=0, \tau=0.0496)$, Fig. $13(\lambda=1, \tau=0.00496)$. These results have been obtained by means of the implicit finite difference scheme with $\sigma=1$ and the method of lines.

\section{The Stationary Solutions of Equations (1.6)}

In $[6,7]$ we obtained the stationary solution of $(1.6),(1.7)$ using the method of stationarity in following way:

$$
\left\{\begin{array}{l}
\frac{\partial p^{(s+1)}}{\partial x}=F_{1}\left(p^{(s)}, f^{(s)}\right) \\
\frac{f^{(s+1)}-f^{(s)}}{\tau}=\mathfrak{L} f^{(s+1)}+F_{2}\left(\omega\left\langle p^{(s+1)}\right\rangle+(1-\omega)\left\langle p^{(s)}\right\rangle\right)
\end{array}\right.
$$

where $s=0,1, \ldots, S$ is the parameter of iterations, $p^{(0)}=\exp \left(i \theta_{0}\right), f^{(0)}=$ $f_{0}(x), S$ is the number of iterations, $\omega \leq 1$ is the positive under relaxation factor. The number $S$ is determined from the following condition: $\max \mid f^{(S)}-$ $f^{(S-1)} \mid \leq 10^{-4}$. For discretization in space nonuniform grid is used with the grid points as the roots of the Chebyshev polynomials of the second kind. For approximation of partial derivatives with respect to $x$ the matrices of derivatives are used. Calculations with $f_{0}(x)=0.12 \sin (\pi x / L), L=15, \tau=1, \delta=0$, $\Delta=0.5, I=0.01, \omega=0.1, \gamma=0.5, K=40, S \approx 500$ result in the optimal efficiency $\eta=0.7288$.

We used MATLAB solvers "ode45, ode15s" for modified $(6.1)(\tau=\infty)$

$$
\left\{\begin{array}{l}
\frac{\partial p^{(s+1)}}{\partial x}=F_{1}\left(p^{(s+1)}, f^{(s)}\right), \\
\mathfrak{L} f^{(s+1)}+F_{2}\left(\omega\left\langle p^{(s+1)}\right\rangle+(1-\omega)\left\langle p^{(s)}\right\rangle\right)=0
\end{array}\right.
$$

where $p^{(0)}=p^{(1)}, f^{(0)}=f_{0}(x), \omega=1 ; 0.5$. The previous results can be obtained with $S \approx 15$. In real calculations with $L=113.7, \omega=1, N=536$ 


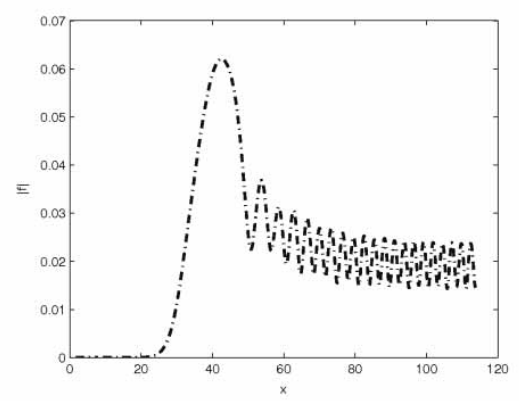

Figure 14. Stationary solution $|f|$ (new), $L=113.7, N=536$.

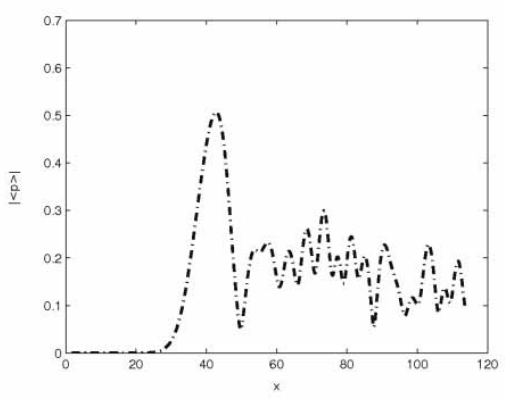

Figure 16. Distribution $|\langle p\rangle|$ (new), $L=113.7, N=536$.

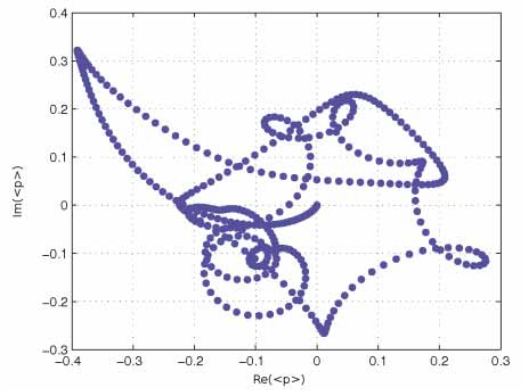

Figure 15. Distribution $\operatorname{Re}(\langle p\rangle), \operatorname{Im}(\langle p\rangle)$ (new), $L=113.7, N=536$.

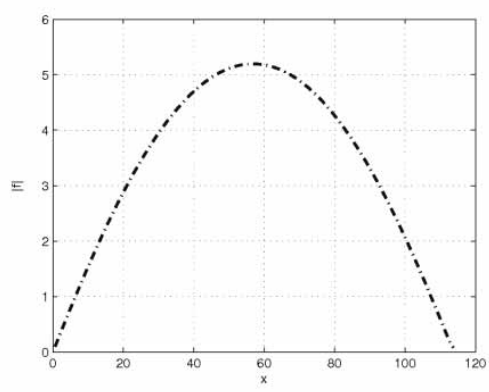

Figure 17. Stationary solution $|f|$ (old), $L=113.7, N=268$.

and $I=0.002249$ we obtained the following distributions of stationary RF field amplitude $|f|$, averaged electron momentum $\langle p\rangle$ in the plane $\operatorname{Re}(\langle p\rangle), \operatorname{Im}(\langle p\rangle)$ and $|\langle p\rangle|$ depending on $x$ :

1. for new version $\eta=0.5860, \max |f|=0.0638$ (see Fig. 14, Fig. 15, Fig. 16) (for boundary conditions $O(h): \eta=0.5862, \max |f|=0.0635$ ),

2. for old version $\eta=0.1610, \max |f|=5.1979$ (see Fig. 17, Fig. 18, Fig. 19) (for $N=268: \max |f|=5.1984$; for $N=134$ : $\max |f|=5.1962$ ).

The boundary value problem for RF field equation is also solved with MATLAB solver "bvp4c", using cubic spline approximation for $\langle p\rangle, g_{b}(x), g_{c}(x), g_{d}(x)$ and initial value of $f_{0}$. The results did not change within three decimal digits.

\section{The Nonstationary Solutions of Equations (1.6)}

For the numerical experiment with the real initial function $f_{0}(x)=0.12 \sin \frac{\pi x}{L}$ computations were performed by means of MATLAB with solvers "ode15", "ode45" for $L=15, \gamma=1.193498, K=25, N=25, I=0.002249, t_{f}=1$, $\delta_{\omega}=0.046565$ and $\Delta=0.538374$. 


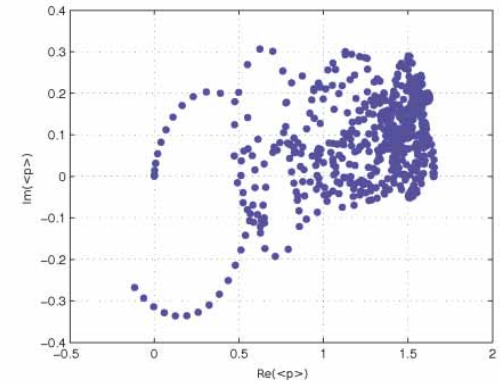

Figure 18. Distribution $\operatorname{Re}(\langle p\rangle), \operatorname{Im}(\langle p\rangle)$ (old), $L=113.7, N=268$.

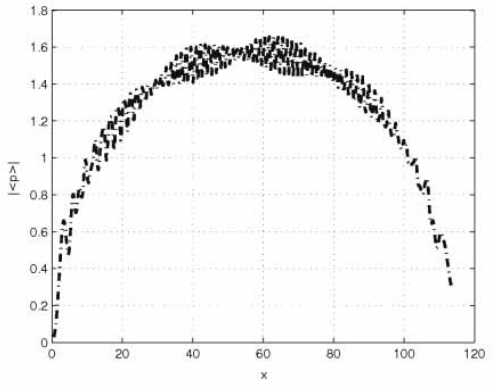

Figure 19. Distribution $|\langle p\rangle|$ (old), $L=113.7, N=268$.

The results of calculations for the efficiency $\eta$ are the following: 0.4854 for method of lines and with all MATLAB solvers (relative error $10^{-6}$ ); for finite difference scheme with $\sigma=1: 0.4808(\tau=0.15), 0.4800(\tau=0.075)$.

Table 3. The values of $\eta, \max \left|f\left(t_{f}, x\right)\right|,\left|f\left(t_{f}, L\right)\right|$ by $L=113.7, \gamma=1.193498, \sigma=1$.

\begin{tabular}{lllllll}
\hline$\sigma=1$ & $\tau=1$ & $\tau=0.5$ & $\tau=0.25$ & $\tau=0.125$ & $\tau=0.1$ & $\begin{array}{l}\text { Method } \\
\text { of lines }\end{array}$ \\
\hline$\eta$ & .4625 & .5056 & .5523 & .5359 & .5277 & .5254 \\
$\max \left|f\left(t_{f}, x\right)\right|$ & .0982 & .0982 & .0982 & .0982 & .0982 & .0982 \\
$\left|f\left(t_{f}, L\right)\right|$ & .0314 & .0319 & .0323 & .0325 & .0326 & .0328 \\
\hline
\end{tabular}

For the nonstationary problem with complex initial function $f_{0}(x), N=$ 134, $K=25, L=113.7$ and $t_{f}=10$ we used the finite difference scheme with $\sigma=1$ and $\sigma=0.5$ and the method of lines (at each automatic step size $\tau$ ). In solving equations (2.1) and (2.3) with the MATLAB we solved the first equation (1.6) with the solver "ode45" with automatic step size and cubic spline interpolation for discrete values of $f$. The solution $p$ is obtained for a fixed time $t$ at uniform grid points $x_{j}$. In the Tab. 3, Tab. 4 we show values of $\eta, \max _{x}\left|f\left(t_{f}, x\right)\right|,\left|f\left(t_{f}, L\right)\right|$ for $\tau=1 ; 0.5 ; 0.25 ; 0.125 ; 0.1$ and $\sigma=1$, $\sigma=0.5$. The calculations with $\sigma=1 / 4$ are unstable. We can see that the value $\max _{x}\left|f\left(t_{f}, x\right)\right|$ does not depend on $\tau$ if $\sigma=1$ and decreases if $\sigma=0.5$ (3.2).

Table 4. The values of $\eta, \max \mid\left(f\left(t_{f}, x\right)|| f,\left(t_{f}, L\right) \mid\right.$ by $L=113.7, \gamma=1.193498, \sigma=0.5$.

\begin{tabular}{lccccc}
\hline$\sigma=0.5$ & $\tau=1$ & $\tau=0.5$ & $\tau=0.25$ & $\tau=0.125$ & $\tau=0.1$ \\
\hline$\eta$ & .5234 & .5036 & .5196 & .5265 & .5225 \\
$\max \left|f\left(t_{f}, x\right)\right|$ & .0986 & .0984 & .0983 & .0983 & .0982 \\
$\left|f\left(t_{f}, L\right)\right|$ & .0339 & .0330 & .0328 & .0328 & .0328 \\
\hline
\end{tabular}

In a number of papers $[3,8,9]$ accurate discretization of Crank-Nicolson scheme $(\sigma=1 / 2)$ has been studied in relation to time dependent Schrödinger 


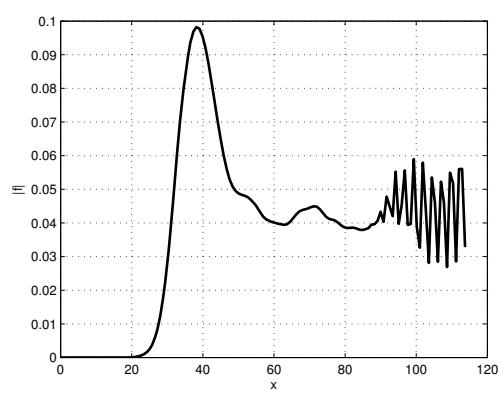

Figure 20. Finite difference scheme solutions for $\left|f\left(t_{f}, x\right)\right|, t_{f}=10$.

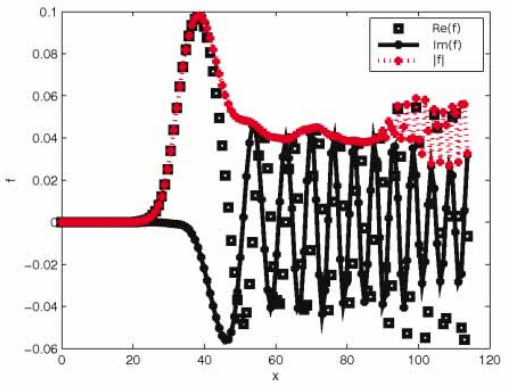

Figure 21. MATLAB solution for $\left.\left|f\left(t_{f}, x\right)\right|, \operatorname{Re}\left(f\left(t_{f}, x\right)\right), \operatorname{Im} f\left(t_{f}, x\right)\right), t_{f}=10$.

type differential equations with different artificial boundary conditions, which guarantee second order temporal approximation and are unconditionally stable.

In [19] it is emphasized that this scheme in solving Schrödinger type equations can be only conditionally temporarily stable, if the asymptotical stability condition is not valid for $t \rightarrow \infty$. Since in gyrotron equation calculations long temporal intervals are considered before the solution becomes stationary, it is more convenient to use implicit difference scheme with $\sigma=1$, because it is also asymptotically stable.

In both cases it can be shown [19], that for a fixed function $\langle p\rangle \in L_{2}$ for fixed $t$ the discrete solution $\mathbf{f}_{h}$ from (3.11) with condition (3.1) approaches the continuous solution $\mathbf{f}$ if $\tau, h \rightarrow 0$, because accuracy $\mathbf{z}_{h}=\mathbf{f}_{h}-\mathbf{f}$ can be estimated as $\left\|\mathbf{z}_{\mathbf{h}}\right\| \leq C\left(\tau^{\alpha}+h^{2}\right)$, where $\alpha=1(\sigma=1), \alpha=2(\sigma=1 / 2)$. In the special case when $\delta_{1}=1, g_{1}=I=0, \gamma=\infty$ (boundary condition of first kind) [19] it has been proved that $\left\|\mathbf{z}_{h}\right\| \leq C\left(\tau^{2}+h^{4}\right)$, if $\sigma=0.5-i h^{2} / 12 \tau$.

We have for $\sigma=0.5$ the following values of $\eta$ : $0.5351(\tau=0.125), 0.5336$ $(\tau=0.1)$. Fig. 20 and Fig. 21 show the discrete functions $\left|f\left(t_{f}, x\right)\right|$ and $\operatorname{Re}\left(f\left(t_{f}, x\right)\right), \operatorname{Im}\left(f\left(t_{f}, x\right)\right),\left|f\left(t_{f}, x\right)\right|$, depending on $x$ as obtained with the finite difference scheme $(\tau=0.1, \sigma=1)$ and MATLAB solvers. The calculation with MATLAB program and method of lines lasts six times longer than implicit calculations with finite difference scheme.

\section{Conclusions}

In the present work the stationary and nonstationary problems of single mode gyrotron equations are investigated. The implicit finite difference schemes and the method of lines are realized with MATLAB. Two versions of gyrotron equations are considered and it is proved that the discrete spatial approximation satisfies the energy conservation law. The results of numerical experiments can be summarized as follows:

- Spectral representations of new and old gyrotron equations are different;

- Method of lines with spatial discretization is an effective algorithm when using MATLAB with sparse matrixes; 
- The implicit finite difference schemes are stable for solving gyrotron equations. It is not useful to employ the three stencil boundary conditions, because the discrete conservation equation holds only approximately;

- New version of gyrotron equation requires long computation times in order to observe oscillations. The full implicit finite difference scheme $(\sigma=1)$ can be used in calculations for long time intervals in those cases when the solution becomes stationary;

- Oscillations are pronounced in the solutions of new equations and are absent in the solutions of old equations (compare Fig. 14 and Fig. 17). The use of numerical algorithms with a variable integration step length and specified accuracy is preferable. Commercially available program packages can be utilized.

\section{Acknowledgments}

One of us (O.D.) expresses his deep gratitude to K.A. Avramides, S. Kern and G.S. Nusinovich for useful discussions regarding the new version of gyrotron equations.

\section{References}

[1] M.I. Airila, O. Dumbrajs, A. Reinfelds and U. Strautiņš. Nonstationary oscillations in gyrotrons. Phys. Plasmas, 8(10):4608-4612, 2001. http://dx.doi.org/10.1063/1.1402173.

[2] M.I. Airila, O. Dumbrajs, A. Reinfelds and D. Teychenné. Traces of stochasticity in electron trajectories in gyrotron resonators. Int. J. Infrared Millimeter Waves, 21(11):1759-1778, 2000. http://dx.doi.org/10.1023/A:1006723600951.

[3] X. Antoine, A. Arnold, C. Besse, M. Ehrhardt and A. Schädle. A review of transparent and artifical boundary conditions techniques for linear and nonlinear Schrödinger equations. Commun. Comput. Phys., 4(4):729-796, 2008.

[4] A. Arnold, M. Ehrbardt and I. Sofronov. Discrete transparent boundary conditions for the Schrödinger equation: fast calculation, approximation, and stability. Commun. Math. Sci., 1(3):501-556, 2003.

[5] K.A. Avramides, O. Dumbrajs, J.L. Vomvoridis and S. Kern. Gyrotron interaction simulations with tapared magnetostatic field. In Conference Proceedings, 35th International Conference on Infrared Millimeter $\mathrm{THz}$ Wawes, Rome, Italy, September 5-10, 2010, p. 17, 2010.

[6] J. Cepītis, H. Kalis and A. Reinfelds. Certain stationary boundary value problem for modelling of gyrotron: numerical investigations. In J. Krope, S.H. Sohrab and F.-K. Benra(Eds.), Theoretical and Experimental Aspects of Heat and Mass Transfer, Proceedings of the 5th WSEAS International Conference on Heat and Mass Transfer (HMT'08), Acapulco, Mexico, January 25-27, 2008, pp. 174-178. WSEAS Press, 2008. 
[7] J. Cepitis, H. Kalis and A. Reinfelds. Numerical investigations of single mode gyrotron equation. Math. Model. Anal., 14(2):169-178, 2009. http://dx.doi.org/10.3846/1392-6292.2009.14.169-178.

[8] R. Čiegis, I. Laukaitytė and M. Radziunas. Numerical algorithms for Schrödinger equation with artifical boundary conditions. Numer. Funct. Anal. Optim., 30(9):903-923, 2009. http://dx.doi.org/10.1080/01630560903393097.

[9] R. Čiegis and M. Radziunas. Effective numerical integration of travelling wave model for edge-emitting broad-area semiconductor lasers and ampfliers. Math. Model. Anal., 15(4):409-430, 2010.

http://dx.doi.org/10.3846/1392-6292.2010.15.409-430.

[10] O. Dumbrajs, H. Kalis and A. Reinfelds. Analysis of difference schemes in modelling of gyrotron equation. Transport and Telecommunication, 5(1):206-214, 2004 .

[11] O. Dumbrajs, H. Kalis and A. Reinfelds. Numerical solution of single mode gyrotron equation. Math. Model. Anal., 9(1):25-38, 2004.

http://dx.doi.org/10.1080/13926292.2004.9637239.

[12] O. Dumbrajs, R. Meyer-Spasche and A. Reinfelds. Analysis of electron trajectories in a gyrotron resonator. IEEE Trans. Plasma Sci., 26(3):846-853, 1998. http://dx.doi.org/10.1109/27.700850.

[13] O. Dumbrajs and A. Reinfelds. Electron trajectories in a realistic gyrotron resonator. Math. Model. Anal., 3:74-80, 1998. http://dx.doi.org/10.1080/13926292.1998.9637088.

[14] M. Erhhardt. Discrete transparent boundary conditions for general Schrödingertype equation. VLSI Design, 4(4):325-338, 1999. http://dx.doi.org/10.1155/1999/72984.

[15] N.S. Ginzburg, G.S. Nusinovich and N.A. Zavolsky. Theory of non-stationary processes in gyrotrons with low Q resonators. Int. J. Electronics, 61(6):881-894, 1986. http://dx.doi.org/10.1080/00207218608920927.

[16] N.S. Ginzburg, N.A. Zavolsky, G.S. Nusinovich and A.S. Sergeev. Onset of auto oscillations in electron microwave generators with diffraction radiation output. Izv. Vyssh. Uchebn. Zaved. Radiofiz., 29(1):106-114, 1986. (in Russian)

[17] G.S. Nusinovich. Introduction to the Physics of Gyrotrons. The Johns Hopkins University Press, Baltimore and London, 2004.

[18] M. Radžiunas and F. Ivanauskas. The stability conditions of finite difference schemes for Schrödinger, Kuramoto-Tszuki and heat equations. Math. Model. Anal., 3:177-194, 1998. http://dx.doi.org/10.1080/13926292.1998.9637101.

[19] A.A. Samarskii. The Theory of Difference Schemes. Marcel Dekker Inc., New York, Basel, 2001. 